\title{
Scar dechannelling limits amount of ablation needed to treat VT
}

Conducting channels are areas of scar tissue that can function as re-entry circuits for ventricular tachycardia (VT). Scar dechannelling-a new technique that targets the entrance of these conducting channels-can limit the amount of ablation required to treat VT and improves outcomes in patients with this arrhythmia.

Investigators enrolled 101 patients scheduled to receive left ventricular ablation for scar-related VT. Those with VT owing to a reversible cause were excluded from the study. Patients were followed up for 21 months (interquartile range 11-29 months).

The team first performed substrate mapping to identify scar-related conducting channels, which were then eliminated by radiofrequency ablation. To ensure all conducting channels had been targeted, the left ventricle was remapped and any remaining channels subjected to a second ablation. The team then induced
VT and performed a final ablation if required to eliminate any residual VT sites.

Patients who required only scar dechannelling had fewer radiofrequency ablations $(19 \pm 11$ versus $27 \pm 18 ; P=0.01)$ and a shorter procedure time $(213 \pm 64 \mathrm{~min}$ versus $244 \pm 71 \mathrm{~min} ; P=0.027)$ than those who required additional VT ablation. Importantly, patients who received scar dechannelling only had better event-free survival (80\% versus $62 \%$ ) and lower mortality ( $5 \%$ versus $11 \% ; P=0.013$ for both outcomes) than those who required additional VT ablation. The investigators conclude that "scar dechanneling alone results in low recurrence [rates] and mortality ... in more than half of patients despite the limited ablation extent required".

Tim Geach

Original article Berruezo, A. et al. Scar dechanneling: a new method for scar-related left ventricular tachycardia substrate ablation. Circ. Arrhythm. Electrophysiol. doi:10.1161/CIRCEP.114.002386 